\title{
Structural and evolutionary characteristics of dynamin-related GTPase OPA1
}

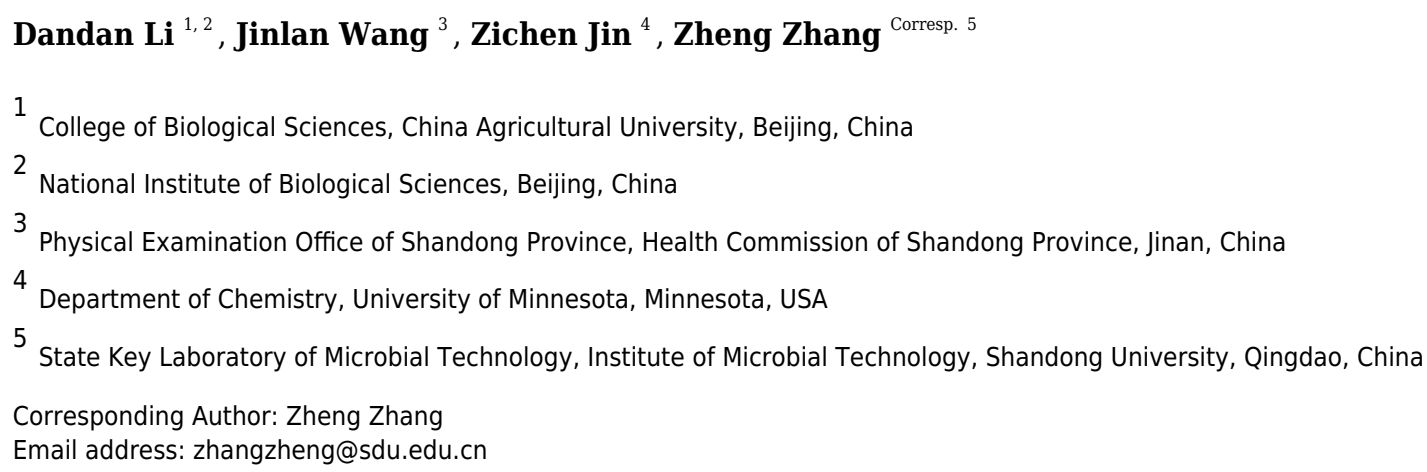

OPA1 is a dynamin-related GTPase that controls mitochondrial fusion, cristae remodeling, energetics and mtDNA maintenance. However, the molecular architecture of OPA1 is poorly understood. Here we modeled the structure of human OPA1 by the threading approach. We found that the C-terminal region of the OPA1 protein had multiple functional domains, while the N-terminal region was rich in alpha helices and did not include specific domains. For the short soluble forms of OPA1, we observed that there were obvious hydrophobic regions near the two cleavage sites and the N-terminal was positively charged after cleavage. The blue native analysis revealed that the protein could form stable homodimers. In addition, the evolutionary conservation of the C-terminal region, where most of the known mutated disease-related sites were located in, was significantly higher than that of the $\mathrm{N}$-terminal region. These findings provided new insights into the structure and biochemical function of OPA1. 
1

2

3

4

5

$6 \quad{ }^{1}$ College of Biological Sciences, China Agricultural University, Beijing 100083, China.

$7 \quad{ }^{2}$ National Institute of Biological Sciences, Beijing 102206, China.

8 3Physical Examination Office of Shandong Province, Health Commission of Shandong Province,

9 Jinan 250014, China.

$10{ }^{4}$ Department of Chemistry, University of Minnesota, Minnesota 55455, USA.

$11{ }^{5}$ State Key Laboratory of Microbial Technology, Institute of Microbial Technology, Shandong

12 University, Qingdao 266237, China.

13

$14 *$ The corresponding author.

15

16 Email addresses of the authors:

17 Dandan Li, lidandan@nibs.ac.cn

18 Jinlan Wang, jinlan726@163.com

19 Zichen Jin, jinxx631@umn.edu

20 Zheng Zhang, zhangzheng@sdu.edu.cn
GTPase OPA1 


\section{Abstract}

22 OPA1 is a dynamin-related GTPase that controls mitochondrial fusion, cristae remodeling,

23 energetics and mtDNA maintenance. However, the molecular architecture of OPA1 is poorly

24 understood. Here we modeled the structure of human OPA1 by the threading approach. We

25 found that the C-terminal region of the OPA1 protein had multiple functional domains, while the

26 N-terminal region was rich in alpha helices and did not include specific domains. For the short

27 soluble forms of OPA1, we observed that there were obvious hydrophobic regions near the two

28 cleavage sites and the N-terminal was positively charged after cleavage. The blue native analysis

29 revealed that the protein could form stable homodimers. In addition, the evolutionary

30 conservation of the C-terminal region, where most of the known mutated disease-related sites

31 were located in, was significantly higher than that of the N-terminal region. These findings

32 provided new insights into the structure and biochemical function of OPA1. 


\section{Introduction}

Mitochondria undergo a constant dynamic balance between organelles fusion and fission to maintain their network morphology and functions (van der Bliek et al. 2013; Wai \& Langer 2016). The functional relevance of mitochondrial dynamics has been emphasized by its requirement during embryonic development and its repercussions in the main functions of the organelle, including respiration, response to cellular stress, calcium homeostasis and apoptosis (Chan 2012; Eisner et al. 2018; Noguchi \& Kasahara 2018). Several conserved GTPase mediate the mitochondrial dynamics: mitofusins (mitofusin 1 and mitofusin 2) control fusion of the outer mitochondrial membrane (OMM) while Drp1 is involved in fission of OMM (Labbe et al. 2014; Pernas \& Scorrano 2016); the conserved dynamin-related GTPase optic atrophy 1 (OPA1) is indispensable for both cristae morphology and the inner mitochondrial membrane (IMM) fusion (Belenguer \& Pellegrini 2013; Del Dotto et al. 2018a; Pernas \& Scorrano 2016).

47 functions, including mitochondrial DNA (mtDNA) maintenance, which is probably anchored to 48 the IMM, and the oxidative phosphorylation efficiency (Belenguer \& Pellegrini 2013; Del Dotto 49 et al. 2018a). In mouse embryonic fibroblasts, global loss of OPA1 results in fragmentation of 50 the mitochondrial network, together with critically reduced mtDNA copy number, as well as 51 significant disorganization of cristae structure and a reduction of respiratory capacity (Chen et al. 52 2010; Cogliati et al. 2013; Song et al. 2007; Song et al. 2009). Re-expression of any OPA1 53 isoform can restore energetic efficiency, mtDNA content, and cristae structure (Del Dotto et al. 
54 2017). OPA1 oligomerization, tightening cristae junctions, is required for apoptosis regulation by 55 maintaining the cytochrome c inside the cristae and controlling its release (Frezza et al. 2006;

56 Olichon et al. 2003; Yamaguchi et al. 2008). In addition, OPA1-dependent modulation of cristae

57 structure is necessary for cellular adaptation to energy substrate availability (Patten et al. 2014). neuropathy that is characterized by selective degeneration of retinal ganglion cells and classically presents in early childhood with progressive visual failure (Del Dotto et al. 2018b; Lenaers et al. 2012). Biochemical studies indicated that OPA1 disease alleles associated with DOA displayed a variety of mitochondrial defects in several activities, involving GTP hydrolysis, cardiolipin association, and membrane tubulation (Ban et al. 2010; Belenguer \& Pellegrini 2013; Zanna et al. 2008). Compared with the classical optic atrophy, DOA and deafness (DOAD) associating neurosensory deafness and DOA plus involving in other clinical manifestations like myopathy, progressive external ophthalmoplegia or spastic paraplegia, are syndromic dominant optic atrophy (Amati-Bonneau et al. 2008; Del Dotto et al. 2018b; Lenaers et al. 2012; Yu-Wai-Man et al. 2010). Moreover, $O P A 1$ mutations have been related to an expanding spectrum of neurodegenerative phenotypes, such as Behr-like syndrome, syndromic parkinsonism, dementia and others (Carelli et al. 2015; Del Dotto et al. 2018b; Marelli et al. 2011). including classical dynamins (dynamin 1, dynamin 2, dynamin 3), mitofusins, Drp1, OPA1, Mx proteins, guanylate-binding proteins (GBPs) and atlastins in eukaryotic cells (Jimah \& Hinshaw 
74 2019). The mitofusins, Drp1, OPA1 and atlastins are involved in the process of membrane

75 remodeling; the classical dynamins mainly function in the clathrin-mediated endocytosis and

76 budding of vesicles; additional dynamins like GBPs and Mx proteins restrict pathogens

77 (Anderson et al. 1999; Haller et al. 1981; Pernas \& Scorrano 2016). Several structures of dynamins have been solved so far (Jimah \& Hinshaw 2019). The structures show that all dynamins include a GTPase domain that binds and hydrolyzes GTP and an $\alpha$-helical bundle domain. Most of dynamins also contain a middle domain involved in oligomerization and a GTPase effector domain (GED) that are associated with stimulation of GTPase activity. Several dynamins contain a domain that can be a transmembrane domain, a sequence or a pleckstrinhomology domain (PH domain) for interacting with lipid membranes (Praefcke \& McMahon 2004). However, due to its exceptional complexity there is still no crystal structure of OPA1 protein. eight mRNA variants by the alternative splicing of exons $4,4 \mathrm{~b}$ and $5 \mathrm{~b}$ (Fig. 1A). OPA1 is a dynamin-related GTPase with a mitochondrial targeting sequence (MTS), followed by a transmembrane (TM), which need to be further cleaved to execute mitochondrial function (Belenguer \& Pellegrini 2013). Precursors translated from the eight OPA1 mRNA are targeted to mitochondria via MTS, which is cleaved by the mitochondrial processing peptidase (MPP) to give rise to the long forms (1-forms) anchored to IMM. About half of 1-forms are then further proteolytically processed by OMA1 and YME1L proteases to produce the short forms (s-forms), 
94 which are soluble in the mitochondrial intermembrane space (IMS) (Anand et al. 2014; Ishihara

95 et al. 2006; MacVicar \& Langer 2016; Song et al. 2007). Remarkably, the four isoforms

96 including exon $4 \mathrm{~b}$ are completely processed into s-forms (Song et al. 2007). Although OPA1

97 protein have been studied for many years, the molecular architecture of OPA1 must be

98 elucidated to better understand its functions. Here, we modeled the structure of the human OPA1

99 by the threading approach, analyzed the protein-protein interaction, and explored the

100 characteristics of the sequences and structures through bioinformatics analysis.

101

\section{Materials \& Methods}

103 OPA1 information acquisition

The information of human $O P A 1$ gene, exons and isoforms were obtained from the NCBI

and Ensemble databases (Zerbino et al. 2018). The other vertebrate OPA1 protein sequences

were acquired from the NCBI reference sequence (RefSeq) database (O'Leary et al. 2016). All

partial sequences and low quality proteins were excluded. The crystal structures of proteins from

dynamin family were obtained from the PDB database (Rose et al. 2017).

109

110

111

112

\section{Sequence analysis}

The theoretical isoelectric points and the grand average hydropathicity were calculated by ExPASy server (Bjellqvist et al. 1993). The eight human OPA1 isoforms and all vertebrates OPA1 proteins were aligned with MAFFT (Katoh \& Standley 2013), respectively. Using the 
113 longest splice variant OPA1 in vertebrates, the evolutionary conservations of amino acid residue

114 positions in the OPA1 sequences was measured by using ConSurf algorithm (Ashkenazy et al.

115 2016). The best evolutionary substitution model was used and calculation was based on the

116 Empirical Bayesian paradigm. The sequence and modeled structure of human OPA1 were used

117 to show the nine-color conservation grades. Sequence logos were generated as graphical

118 representations of the multiple sequence alignments of the amino acids (Crooks et al. 2004).

119 Phylogenetic analyses were conducted using MEGA version 7 by the bootstrap neighbor joining

120 method (Kumar et al. 2016).

121 Structural analysis

123 The structural model was refined by fragment-guided molecular dynamics (FG-MD) simulations

124 at the atomic-level (Zhang et al. 2011). The quality assessment of Ramachandran plot had been

125 used to quantitatively assess the accuracy of protein structure predictions. The statistical data of

126 Ramachandran plot was calculated by PROCHECK (Laskowski et al. 1993). The protein

127 structures were displayed by PyMol (Schrödinger, LLC). Pairwise structural differences between

128 human OPA1 and other proteins from dynamin family were measured by TM-align (Zhang \&

129 Skolnick 2005). The structural similarity of two protein structures was measured with the TM-

130 score that had the value $(0,1)$ ( Xu \& Zhang 2010). The higher the value was, the more similar

131 the two aligned structures were. The two modeled structures of human OPA1 protein were taken

132 as target 1 and target 2, respectively, to submit to PRISM protein-protein docking server for 
133 predicting possible interactions and how the interaction partners structurally connect (Baspinar et

134 al. 2014).

135

136

137

138

139

140

141

142

143

144

145

146

147

148

149

150

151

152

\section{Protein purification and blue native page}

A human OPA1 construct (exons 6-28) was expressed in Escherichia coli BL21 (DE3) cells as $\mathrm{N}$-terminal $\mathrm{His}_{6}$-SUMO-tagged fusion proteins using the pET28a plasmid (His 6 -SUMOOPA1). Cells were grown in $\mathrm{LB}$ medium at $37^{\circ} \mathrm{C}$ to an $\mathrm{OD}_{600}$ of $\sim 0.6$. Protein expression was induced by addition of $100 \mu \mathrm{M}$ IPTG and cultures were incubated overnight at $16{ }^{\circ} \mathrm{C}$. The cell pellet was resuspended in $10 \mathrm{mM}$ Tris- $\mathrm{HCl} \mathrm{pH} 8.0,20 \mathrm{mM}$ imidazole and $500 \mathrm{mM} \mathrm{NaCl}$ buffer. Cells were lysed using a microfluidizer (Microfluidics). After centrifugation at 12,000 rpm for 1 h at $4{ }^{\circ} \mathrm{C}$, the soluble extract was filtered and combined with Ni-NTA agarose (GE Healthcare) in batch. After beads were packed into a column, the combination was washed with resuspension buffer supplemented with $40 \mathrm{mM}$ imidazole, and protein was eluted with an imidazole gradient to a final concentration of $500 \mathrm{mM}$. Peak fractions were pooled, and the His ${ }_{6}$-SUMO-tag was cleaved by ULP1 peptidase in the $4{ }^{\circ} \mathrm{C}$ overnight to generate the OPA1 (exons 6-28) protein. Then diluted the $\mathrm{NaCl}$ concentration to $50 \mathrm{mM}$ and applied to Resource $\mathrm{Q}$ column (GE Healthcare). Peak fractions were concentrated and loaded to a Superdex 200 10/300 gel filtration column (GE Healthcare) using the buffer with $10 \mathrm{mM}$ Tris-HCl, pH 8.0 and $150 \mathrm{mM} \mathrm{NaCl}$. The protein was concentrated to $\sim 10 \mathrm{mg} / \mathrm{ml}$ prior to freezing. All the samples were analyzed by SDSPAGE electrophoresis. The procedure for SDS-PAGE was based on a general protocol as previously described (Simpson 2006). All the samples heated at $95^{\circ} \mathrm{C}$ for $10 \mathrm{~min}$ and loaded onto 
153 a polyacrylamide gel that was consist of 5\% stacking and $10 \%$ resolving gel, which was run at 80

$154 \mathrm{~V}$ for $0.5 \mathrm{~h}$, and then at $100 \mathrm{~V}$ for $1 \mathrm{~h}$.

155

Blue native page was performed as described previously with slight modifications (Wittig et

156

157

158

159

160

161

162

163

164

165

166

167

168

169

170

171

al. 2006). $10 \mathrm{mg}$ of the OPA1 (exons 6-28) protein was loaded into a blue native page gel that was consist of $3.5 \%$ stacking and $4-10 \%$ gradient separating gel. The cathode buffer $(7.5 \mathrm{mM}$ imidazole with $\mathrm{pH} 7.0,50 \mathrm{mM}$ tricine, supplemented with $0.02 \% \mathrm{w} / \mathrm{v}$ coomassie brilliant blue G250) and the anode (25 mM imidazole with $\mathrm{pH} 7.0)$ were chilled at $4{ }^{\circ} \mathrm{C}$ before used. Then, electrophoresis was started at $80 \mathrm{~V}$ for $30 \mathrm{~min}$ and adjusted to $120 \mathrm{~V}$ for $10 \mathrm{~h}$.

\section{Results}

\section{Sequence analysis of human OPA1 isoforms}

The human OPA1 gene contains eight spliced variants (Fig. 1A). Proteolytically processed by OMA1 and YME1L proteases in the domains were corresponding to exons 5 and $5 b$, containing the cleavage sites $\mathrm{S} 1$ and $\mathrm{S} 2$, respectively. In principle, each mRNA variant form can produce a long isoform and one or more short isoforms. However, the four isoforms $(3,5,6,8)$ including exon $4 \mathrm{~b}$ completely processed into s-forms. We confirmed that exon $4 \mathrm{~b}$-encoded peptide was hydrophobic while the others around were hydrophilic (Fig. 1B), which was consistent with the previous study (Elachouri et al. 2011). The hydrophobicity of exon 4bencoded peptide might be helpful to recruit the OMA1 protease, thus, promoting the cleavage at 
172 site S1. Although exon 5b-encoded peptide was hydrophilic as a whole, there was a hydrophobic

173 region just before site S2, hypothesizing that the hydrophobic segment can associate with

174 YME1L protease to promote the cleavage at site S2.

175 We also noticed that the N-terminus of OPA1 s-forms were positively charged (Fig. 1C).

176 The isoelectric point $(\mathrm{pI})$ value of exon 5-encoded peptide was 6.8 , while the pI value of the

177 remaining peptide segment increased to 8.6 after being cleaved at site S1. Similarly, the pI value

178 of exon 5 b-encoded peptide rose from 6.8 to 9.5 after the site S2 was cut. This result revealed

179 that the N-terminal of OPA1 s-forms all had positive charge no matter it was cleaved at site S1 or 180 S2.

\section{Structure characteristic of human OPA1}

Taking the longest splice variant 8 including 30 exons as an example, we used the threading approach to model the three-dimensional structure of human OPA1 protein excluding MTS and TM regions (Fig. 2). According to quality assessment, the accuracy of the OPA1 protein structural models were acceptable (Fig. S1). The structure showed that OPA1 protein could be clearly divided into two regions, the $\mathrm{N}$-terminal and $\mathrm{C}$-terminal region. The $\mathrm{N}$-terminal region (encoded by exons 3-8) was rich in alpha helix, which did not include specific domains. The difference among the 8 spliced variants of OPA1 protein, l-forms and s-forms was the region of $\mathrm{N}$-terminal. The N-terminal region of 1-forms contained 138-228 amino acid residues, while sforms included only 104-134 residues. 
The C-terminal region of OPA1 protein was a dense structure containing multiple domains

193

194

195

196

197

198

199

200

201

202

203

204

205

206

207

208

209

210

211

and was identical for all isoforms. In the human OPA1 protein, each domain of the C-terminal

region had perfectly corresponded with exons. The GTPase domain corresponded with exons 9-

16, middle domain corresponded with exons 19-22 and a GED corresponded with exons 26-

28. The peptide encoded by exons 17-18 formed a long helix that connected GTPase domain and middle domain. The exons 23-25 encoded a peptide, a PH domain, located between middle domain and GED.

The PH domain of classical dynamins is responsible for their interaction with negatively charged lipid membranes (Praefcke \& McMahon 2004). The pI analysis indicated that the pI value of PH domain of human OPA1 protein was 7.7, especially the pI value of exon 25 -encoded peptide was as high as 9.5 whereas the $\mathrm{pI}$ value of the middle domain and GED next to PH domain were 5.0 and 6.8 , respectively. Thus, the PH domain of OPA1 carried positive charge, might be involved in interaction with negatively charged phospholipid molecules.

Comparing the structure of the C-terminal region of human OPA1 protein with the nineknown structures of the dynamin superfamily proteins (Fig. 3), the results showed that OPA1 and other human dynamins had two common domains: a GTPase domain and an $\alpha$-helical bundle. Interestingly, human OPA1 protein was similar to dynamin 1, dynamin 3, Drp1, MxA and MxB proteins, and the TM-score of all was higher than 0.5 , although these proteins did not mediate membrane fusion like OPA1. Comparing OPA1 with mitofusin 1, atlastin 1 and altastin 3, which were involved in membrane fusion, the TM-score of all was minor than 0.3. In addition, similar 
212 to dynamin 1, dynamin 3 and Drp1, OPA1 had a PH domain, while other proteins involved in

213 fusion process did not have. Significant structural differences indicated that OPA1 might have a

214 unique mechanism for controlling inner membrane fusion.

\section{Dimerization of human OPA1 protein}

217

To determine the oligomeric form of OPA1, we expressed the common part of the eight

218

219

220

221

222

223

224

225

226

227

228

229

230

231

spliced variants of human OPA1 (exons 6-28) protein in E. coli. The protein was detected by SDS-PAGE electrophoresis (Fig. S2). From the peak position of the OPA1 (exons 6-28) elution profiles of a S200 size-exclusion column, the result indicated that the molecular weight of the protein was about $170 \mathrm{kD}$, thus, we speculated the protein may exist as dimers in solution. Additionally, in blue native electrophoresis, we found nucleotide-free OPA1 formed stable dimers and multiple oligomers, indicating that the OPA1 molecule itself could be interacted and formed a stable multimer.

Further, we predicted the possible mode of dimerization of the C-terminal region by using the human OPA1 protein modeled structure. Protein-protein docking results showed that the Cterminal region of the human OPA1 protein could form two stable homodimers modes. In the first mode of homodimer, the GTPase domains of the two monomers were approximately $130 \AA$ apart, and the dimeric interface involved in 23 pairs of interaction among 15 amino acid sites, 10 pairs of which were from the interaction between the linker domain and middle domain, 12 pairs of which were from the interaction between the middle domain and the GED, while one pair of 
232 sites was between the two same GED domains (Fig. 4A). In the second homodimer, the GTPase

233 domains of the two monomers were approximately $100 \AA$ apart, and the dimeric interaction

234 interface involved in 33 pairs of interaction among 18 amino acid sites, 17 pairs of which were

235 from the interaction between two middle domains, 12 pairs of which were from the interaction

236 between the middle domain and the GED, and 4 pairs from the interaction between the two GED

237 domains (Fig. 4B). These results indicated that, similar to dynamin 1 and MxA, OPA1 could

238 assemble to dimers and higher-order oligomers via middle domain and GED, which was

239 different from mitofusins that mediated membrane fusion by GTPase domains (Cao et al. 2017).

240 Additionally, interactions between GTPase-GTPase domains had been reported to be essential

241 for the function of dynamins, but our results showed neither of these two dimeric interfaces of

242 nucleotide-free OPA1 proteins involved in the GTPase domain.

\section{Conserved sites in OPA1 protein}

Furthermore, we collected all the vertebrate OPA1 proteins by sequence similarity search.

Totally, more than 900 of OPA1 protein sequences were discovered from 205 species including several spliced variants. The sequence alignments was shown in the form of sequence logo map (Fig. S3). Using the longest spliced variant of OPA1 protein sequence in each species of vertebrates, we analyzed conserved amino acid sites in the OPA1 proteins and displayed them through the modeled structure of the human OPA1 protein (Fig. 5A). 
253 (grade 9), the N-terminal region only occupied $27 \%$ of all sites and $66 \%$ in the C-terminal region.

254 For those sites with the lowest conservation (grade 1), 43\% of all sites were in the N-terminal 255 region and $10 \%$ in the $\mathrm{C}$-terminal region. Specifically, in the $\mathrm{N}$-terminal region, the exon 4 and exon 5-encoded peptides had the lowest conservation while the peptides encoded by exons 6-8

257 had a higher conservation. In the C-terminal region, the conservation of the GTPase domain and the linker encoded by exons 17-18 were the highest, while the PH domain was less conservative. These results indicated that the linkers encoded by exons 17-18 and exons 6-8, next to the

260 GTPase domain, may be functionally important. A large swing in the linker during the GTPase cycle might cause the power-stroke that led to fusion. However, being the PH domain less conserved, it was speculated that its function might vary from species to species.

\section{Disease-related sites in human OPA1 protein}

Human OPA1 mutations have been associated to a large spectrum of neurodegenerations.

171 amino acid site variants in the human OPA1 had been analyzed by the locus-specific

protein were in accord with those of spliced variant 8. These variations include substitutions, 
271 were not related to diseases and 25 were variants of unknown significance. About $80 \%$ of 272 disease-related variant sites were the highest conservative in the vertebrate OPA1 proteins (grade 2739 ), while only $30 \%$ of the variant sites unrelated to disease had the corresponding values. Besides, $27460 \%$ of variant sites that were not related to diseases had the lowest conservation (grade 1), while 275 less than $10 \%$ of disease-related variant sites were the lowest conservative. Therefore, disease276 related mutations in the OPA1 protein occurred mainly at sites with highly evolutionary 277 conservation. related mutation sites located in this region accounted for only $15 \%$ of the total disease-related mutation sites in the OPA1 while the mutations unrelated to diseases in this region occupied $70 \%$ of the total mutation sites unrelated to diseases. The disease-related variant sites in the Nterminal region were concentrated in the exons $1-2$ coding region ( 8 variants) and the exons $6-8$ coding region (11 variants), while the variant sites unrelated to disease were concentrated on the exons $4,4 \mathrm{~b}$ and $5 \mathrm{~b}$ (5 variants). For the C-terminal region, more than $25 \%$ of the sites in the GTPase domain and the linker region encoded by exons 17-18 were found to have diseaserelated mutations, respectively. And about $20 \%$ of sites in the GED were also found to present related mutation occurred. 
291

292

293

294

295

296

297

298

299

300

301

302

303

304

305

306

307

308

309

310

\section{Discussion}

OPA1 is a member of dynamin superfamily, which is essential for shaping the cristae morphology and IMM fusion (Belenguer \& Pellegrini 2013; Praefcke \& McMahon 2004). In human, the OPA1 proteins include soluble s-forms and 1-forms containing the TM region. However, there is a lack of understanding of the whole structure of OPA1 and the structural differences among various spliced variants, 1-forms and s-forms. In our study, the modeled structure of human OPA1 protein revealed that its structure was divided into N-terminal and Cterminal regions. N-terminal region did not contain the specific domains and was structurally a long peptide chain rich in alpha helices. The length of the long peptide chain in the N-terminal region was the only difference among the eight OPA1 isoforms, while there was no difference in the functional domains. For the OPA1 s-forms, it lacked the TM region and the length of the peptide chain in the N-terminal region was shorten. More interestingly, we found that the Nterminal of exons 5 and $5 \mathrm{~b}$ encoding peptides were positively charged after cleavage, indicating that OPA1 s-forms may interact with the negatively charged phospholipid molecules in the membrane. a middle domain, a PH domain and a GED. The structure of this region was different from mitofusins and atlastins that mediated membrane fusion, while it was similar to dynamin 1 and MxA. The OPA1 protein contained a PH domain that interacted with phospholipid molecules, while mitofusins and atlastins did not include. In addition, similar to dynamin 1 and MxA, OPA1 
311

312

313

314

315

316

317

318

319

320

321

322

323

324

325

326

327

328

329

could self-assemble to form dimers through the middle domain and GED, while this was also different from mitofusins and atlastins that dimerized by GTPase-GTPase domains. All these findings indicated that OPA1 might have a specific fusion mechanism.

The prior studies indicated that a homotypic OPA1 interaction, tightening cristae junctions, mediated membrane tethering (Ban et al. 2017; Frezza et al. 2006; Olichon et al. 2003). Our modeled structural analysis indicated that the C-terminal region of human OPA1 protein could form stable homodimer. Although the obvious difference between the eight OPA1 isoforms was concentrated on the N-terminal region, all of them maybe adopt the same mode by trans-OPA1 interaction when maintaining the cristae morphology. The OPA1 s-forms could also form a trans-interaction, and its $\mathrm{N}$-terminal might interact with cardiolipin in the membrane. An in vitro membrane fusion assay unveiled that OPA1 1-form on one side of the membrane and cardiolipin on the other side, are the minimal components sufficient and necessary for fusion (Ban et al. 2017; Liu \& Chan 2017). According to the electric charge analysis, the PH domain had a strong positive charge region while cardiolipin had a negative charge region. Furthermore, a deletion mutant assay suggested that the domain next to the GTPase domain was necessary for cardiolipin binding (Ban et al. 2017; Del Dotto et al. 2018a). Combined with our work, we speculated that the charge interaction between $\mathrm{PH}$ domain and cardiolipin promoted binding and then accelerated double-membrane fusion. In short, these findings could be useful to better understand the biochemical functions of OPA1. 
331 Conclusions

332

333

334

335

336

337

338

339

340

341

342

343

344

345

346

347

348

349

structure was divided into $\mathrm{N}$-terminal and $\mathrm{C}$-terminal region. The $\mathrm{N}$-terminal region was rich in alpha helices and did not include specific domains. The eight OPA1 spliced variants only differed in the length of the long peptide chain in the N-terminal region but not in the functional domains. By contrast, the C-terminal region of OPA1 protein was a dense structure containing a GTPase domain, a middle domain, a PH domain, and a GED. The structure of the C-terminal region of OPA1 protein was not similar to that of other dynamin superfamily members that mediated membrane fusion, while it was similar to dynamin 1 and MxA, which could selfassembled to form dimers by the middle domain and GED. Additionally, the evolutionary conservation of the $\mathrm{C}$-terminal region was significantly higher than that of the $\mathrm{N}$-terminal region and the known mutated disease-related sites were mostly located in the C-terminal region of OPA1 protein. Overall, these findings provide novel insights into the structural and evolutionary characterizations of OPA1.

\section{Acknowledgements}

We acknowledge Professor Xiangshu Jin of Michigan State University for insight advices on biochemical assays. 
350

351

352

353

354

355

356

357

358

359

360

361

362

363

364

365

366

367

368

\section{References}

Amati-Bonneau P, Valentino ML, Reynier P, Gallardo ME, Bornstein B, Boissiere A, Campos Y, Rivera H, de la Aleja JG, Carroccia R, Iommarini L, Labauge P, Figarella-Branger D, Marcorelles P, Furby A, Beauvais K, Letournel F, Liguori R, La Morgia C, Montagna P, Liguori M, Zanna C, Rugolo M, Cossarizza A, Wissinger B, Verny C, Schwarzenbacher R, Martin MA, Arenas J, Ayuso C, Garesse R, Lenaers G, Bonneau D, and Carelli V. 2008. OPA1 mutations induce mitochondrial DNA instability and optic atrophy 'plus' phenotypes. Brain 131:338-351. 10.1093/brain/awm298

Anand R, Wai T, Baker MJ, Kladt N, Schauss AC, Rugarli E, and Langer T. 2014. The i-AAA protease YME1L and OMA1 cleave OPA1 to balance mitochondrial fusion and fission. $J$ Cell Biol 204:919-929. 10.1083/jcb.201308006

Anderson SL, Carton JM, Lou J, Xing L, and Rubin BY. 1999. Interferon-induced guanylate binding protein-1 (GBP-1) mediates an antiviral effect against vesicular stomatitis virus and encephalomyocarditis virus. Virology 256:8-14. 10.1006/viro.1999.9614

Ashkenazy H, Abadi S, Martz E, Chay O, Mayrose I, Pupko T, and Ben-Tal N. 2016. ConSurf 2016: an improved methodology to estimate and visualize evolutionary conservation in macromolecules. Nucleic Acids Res 44:W344-350. 10.1093/nar/gkw408

Ban T, Heymann JA, Song Z, Hinshaw JE, and Chan DC. 2010. OPA1 disease alleles causing dominant optic atrophy have defects in cardiolipin-stimulated GTP hydrolysis and 
370

371

372

Ban T, Ishihara T, Kohno H, Saita S, Ichimura A, Maenaka K, Oka T, Mihara K, and Ishihara N. 2017. Molecular basis of selective mitochondrial fusion by heterotypic action between OPA1 and cardiolipin. Nat Cell Biol 19:856-863. 10.1038/ncb3560

Baspinar A, Cukuroglu E, Nussinov R, Keskin O, and Gursoy A. 2014. PRISM: a web server and repository for prediction of protein-protein interactions and modeling their 3D complexes. Nucleic Acids Res 42:W285-289. 10.1093/nar/gku397

Belenguer P, and Pellegrini L. 2013. The dynamin GTPase OPA1: more than mitochondria? Biochim Biophys Acta 1833:176-183. 10.1016/j.bbamcr.2012.08.004

Bjellqvist B, Hughes GJ, Pasquali C, Paquet N, Ravier F, Sanchez JC, Frutiger S, and Hochstrasser D. 1993. The focusing positions of polypeptides in immobilized $\mathrm{pH}$ gradients can be predicted from their amino acid sequences. Electrophoresis 14:10231031.

Cao YL, Meng S, Chen Y, Feng JX, Gu DD, Yu B, Li YJ, Yang JY, Liao S, Chan DC, and Gao S. 2017. MFN1 structures reveal nucleotide-triggered dimerization critical for mitochondrial fusion. Nature 542:372-376. 10.1038/nature21077

Carelli V, Musumeci O, Caporali L, Zanna C, La Morgia C, Del Dotto V, Porcelli AM, Rugolo M, Valentino ML, Iommarini L, Maresca A, Barboni P, Carbonelli M, Trombetta C, Valente EM, Patergnani S, Giorgi C, Pinton P, Rizzo G, Tonon C, Lodi R, Avoni P, 
388

389

390

391

392

393

394

395

396

397

398

399

400

401

402

403

404

405

406

Liguori R, Baruzzi A, Toscano A, and Zeviani M. 2015. Syndromic parkinsonism and dementia associated with OPA1 missense mutations. Ann Neurol 78:21-38. 10.1002/ana.24410

Chan DC. 2012. Fusion and fission: interlinked processes critical for mitochondrial health. Annu Rev Genet 46:265-287. 10.1146/annurev-genet-110410-132529

Chen H, Vermulst M, Wang YE, Chomyn A, Prolla TA, McCaffery JM, and Chan DC. 2010. Mitochondrial fusion is required for mtDNA stability in skeletal muscle and tolerance of mtDNA mutations. Cell 141:280-289. 10.1016/j.cell.2010.02.026

Cogliati S, Frezza C, Soriano ME, Varanita T, Quintana-Cabrera R, Corrado M, Cipolat S, Costa V, Casarin A, Gomes LC, Perales-Clemente E, Salviati L, Fernandez-Silva P, Enriquez JA, and Scorrano L. 2013. Mitochondrial cristae shape determines respiratory chain supercomplexes assembly and respiratory efficiency. Cell 155:160-171. 10.1016/j.cell.2013.08.032

Crooks GE, Hon G, Chandonia JM, and Brenner SE. 2004. WebLogo: a sequence logo generator. Genome Res 14:1188-1190. 10.1101/gr.849004

Del Dotto V, Fogazza M, Carelli V, Rugolo M, and Zanna C. 2018a. Eight human OPA1 isoforms, long and short: What are they for? Biochim Biophys Acta Bioenerg 1859:263269. 10.1016/j.bbabio.2018.01.005

Del Dotto V, Fogazza M, Lenaers G, Rugolo M, Carelli V, and Zanna C. 2018b. OPA1: How 
407

408

409

much do we know to approach therapy? Pharmacol Res 131:199-210.

Del Dotto V, Mishra P, Vidoni S, Fogazza M, Maresca A, Caporali L, McCaffery JM, 10.1016/j.phrs.2018.02.018

Cappelletti M, Baruffini E, Lenaers G, Chan D, Rugolo M, Carelli V, and Zanna C. 2017. OPA1 Isoforms in the Hierarchical Organization of Mitochondrial Functions. Cell Rep 19:2557-2571. 10.1016/j.celrep.2017.05.073

Eisner V, Picard M, and Hajnoczky G. 2018. Mitochondrial dynamics in adaptive and maladaptive cellular stress responses. Nat Cell Biol 20:755-765. 10.1038/s41556-0180133-0

Elachouri G, Vidoni S, Zanna C, Pattyn A, Boukhaddaoui H, Gaget K, Yu-Wai-Man P, Gasparre G, Sarzi E, Delettre C, Olichon A, Loiseau D, Reynier P, Chinnery PF, Rotig A, Carelli V, Hamel CP, Rugolo M, and Lenaers G. 2011. OPA1 links human mitochondrial genome maintenance to mtDNA replication and distribution. Genome Res 21:12-20. $10.1101 /$ gr. 108696.110

Frezza C, Cipolat S, Martins de Brito O, Micaroni M, Beznoussenko GV, Rudka T, Bartoli D, Polishuck RS, Danial NN, De Strooper B, and Scorrano L. 2006. OPA1 controls apoptotic cristae remodeling independently from mitochondrial fusion. Cell 126:177-189. 10.1016/j.cell.2006.06.025

Haller O, Arnheiter H, Gresser I, and Lindenmann J. 1981. Virus-specific interferon action. 
426

427

428

429

430

431

432

433

434

435

436

437

438

439

440

441

442

443

444

Protection of newborn Mx carriers against lethal infection with influenza virus. $J$ Exp Med 154:199-203.

Ishihara N, Fujita Y, Oka T, and Mihara K. 2006. Regulation of mitochondrial morphology through proteolytic cleavage of OPA1. EMBO J 25:2966-2977. 10.1038/sj.emboj.7601184

Jimah JR, and Hinshaw JE. 2019. Structural Insights into the Mechanism of Dynamin Superfamily Proteins. Trends Cell Biol 29:257-273. 10.1016/j.tcb.2018.11.003

Katoh K, and Standley DM. 2013. MAFFT multiple sequence alignment software version 7: improvements in performance and usability. Mol Biol Evol 30:772-780. 10.1093/molbev/mst010

Kumar S, Stecher G, and Tamura K. 2016. MEGA7: Molecular Evolutionary Genetics Analysis Version 7.0 for Bigger Datasets. Mol Biol Evol 33:1870-1874. 10.1093/molbev/msw054

Labbe K, Murley A, and Nunnari J. 2014. Determinants and functions of mitochondrial behavior. Annu Rev Cell Dev Biol 30:357-391. 10.1146/annurev-cellbio-101011-155756

Laskowski RA, MacArthur MW, Moss DS, and Thornton JM. 1993. PROCHECK: a program to check the stereochemical quality of protein structures. Journal of applied crystallography 26:283-291.

Lenaers G, Hamel C, Delettre C, Amati-Bonneau P, Procaccio V, Bonneau D, Reynier P, and Milea D. 2012. Dominant optic atrophy. Orphanet J Rare Dis 7:46. 10.1186/1750-1172- 
446

447

448

449

450

451

452

453

454

455

456

457

458

459

460

461

462

463

Liu R, and Chan DC. 2017. OPA1 and cardiolipin team up for mitochondrial fusion. Nat Cell Biol 19:760-762. 10.1038/ncb3565

MacVicar T, and Langer T. 2016. OPA1 processing in cell death and disease - the long and short of it. J Cell Sci 129:2297-2306. 10.1242/jcs.159186

Marelli C, Amati-Bonneau P, Reynier P, Layet V, Layet A, Stevanin G, Brissaud E, Bonneau D, Durr A, and Brice A. 2011. Heterozygous OPA1 mutations in Behr syndrome. Brain 134:e169; author reply e170. 10.1093/brain/awq306

Noguchi M, and Kasahara A. 2018. Mitochondrial dynamics coordinate cell differentiation. Biochem Biophys Res Commun 500:59-64. 10.1016/j.bbrc.2017.06.094

O'Leary NA, Wright MW, Brister JR, Ciufo S, Haddad D, McVeigh R, Rajput B, Robbertse B, Smith-White B, Ako-Adjei D, Astashyn A, Badretdin A, Bao Y, Blinkova O, Brover V, Chetvernin V, Choi J, Cox E, Ermolaeva O, Farrell CM, Goldfarb T, Gupta T, Haft D, Hatcher E, Hlavina W, Joardar VS, Kodali VK, Li W, Maglott D, Masterson P, McGarvey KM, Murphy MR, O'Neill K, Pujar S, Rangwala SH, Rausch D, Riddick LD, Schoch C, Shkeda A, Storz SS, Sun H, Thibaud-Nissen F, Tolstoy I, Tully RE, Vatsan AR, Wallin C, Webb D, Wu W, Landrum MJ, Kimchi A, Tatusova T, DiCuccio M, Kitts P, Murphy TD, and Pruitt KD. 2016. Reference sequence (RefSeq) database at NCBI: current status, taxonomic expansion, and functional annotation. Nucleic Acids Res 
465

466

467

468

469

470

471

472

473

474

475

476

477

478

479

480

481

482

Olichon A, Baricault L, Gas N, Guillou E, Valette A, Belenguer P, and Lenaers G. 2003. Loss of OPA1 perturbates the mitochondrial inner membrane structure and integrity, leading to cytochrome c release and apoptosis. $J$ Biol Chem 278:7743-7746. 10.1074/jbc.C200677200

Patten DA, Wong J, Khacho M, Soubannier V, Mailloux RJ, Pilon-Larose K, MacLaurin JG, Park DS, McBride HM, Trinkle-Mulcahy L, Harper ME, Germain M, and Slack RS. 2014. OPA1-dependent cristae modulation is essential for cellular adaptation to metabolic demand. EMBO J 33:2676-2691. 10.15252/embj.201488349

Pernas L, and Scorrano L. 2016. Mito-Morphosis: Mitochondrial Fusion, Fission, and Cristae Remodeling as Key Mediators of Cellular Function. Annu Rev Physiol 78:505-531. 10.1146/annurev-physiol-021115-105011

Praefcke GJ, and McMahon HT. 2004. The dynamin superfamily: universal membrane tubulation and fission molecules? Nat Rev Mol Cell Biol 5:133-147. 10.1038/nrm1313

Rose PW, Prlic A, Altunkaya A, Bi C, Bradley AR, Christie CH, Costanzo LD, Duarte JM, Dutta S, Feng Z, Green RK, Goodsell DS, Hudson B, Kalro T, Lowe R, Peisach E, Randle C, Rose AS, Shao C, Tao YP, Valasatava Y, Voigt M, Westbrook JD, Woo J, Yang H, Young JY, Zardecki C, Berman HM, and Burley SK. 2017. The RCSB protein data bank: integrative view of protein, gene and 3D structural information. Nucleic Acids Res 
484

485

486

487

488

489

490

491

492

493

494

495

496

497

498

499

500

501

Simpson RJ. 2006. SDS-PAGE of Proteins. CSH Protoc 2006. 10.1101/pdb.prot4313

Song Z, Chen H, Fiket M, Alexander C, and Chan DC. 2007. OPA1 processing controls mitochondrial fusion and is regulated by mRNA splicing, membrane potential, and Yme1L. J Cell Biol 178:749-755. 10.1083/jcb.200704110

Song Z, Ghochani M, McCaffery JM, Frey TG, and Chan DC. 2009. Mitofusins and OPA1 mediate sequential steps in mitochondrial membrane fusion. Mol Biol Cell 20:3525-3532. 10.1091/mbc.E09-03-0252

van der Bliek AM, Shen Q, and Kawajiri S. 2013. Mechanisms of mitochondrial fission and fusion. Cold Spring Harb Perspect Biol 5. 10.1101/cshperspect.a011072

Wai T, and Langer T. 2016. Mitochondrial Dynamics and Metabolic Regulation. Trends Endocrinol Metab 27:105-117. 10.1016/j.tem.2015.12.001

Wittig I, Braun HP, and Schagger H. 2006. Blue native PAGE. Nat Protoc 1:418-428. $10.1038 /$ nprot.2006.62

$\mathrm{Xu}$ J, and Zhang Y. 2010. How significant is a protein structure similarity with TM-score $=0.5$ ? Bioinformatics 26:889-895. 10.1093/bioinformatics/btq066

Yamaguchi R, Lartigue L, Perkins G, Scott RT, Dixit A, Kushnareva Y, Kuwana T, Ellisman MH, and Newmeyer DD. 2008. Opa1-mediated cristae opening is Bax/Bak and BH3 dependent, required for apoptosis, and independent of Bak oligomerization. Mol Cell 
503

504

505

506

507

Yang J, Yan R, Roy A, Xu D, Poisson J, and Zhang Y. 2015. The I-TASSER Suite: protein structure and function prediction. Nat Methods 12:7-8. 10.1038/nmeth.3213

Yu-Wai-Man P, Griffiths PG, Gorman GS, Lourenco CM, Wright AF, Auer-Grumbach M, Toscano A, Musumeci O, Valentino ML, Caporali L, Lamperti C, Tallaksen CM, Duffey P, Miller J, Whittaker RG, Baker MR, Jackson MJ, Clarke MP, Dhillon B, Czermin B, Stewart JD, Hudson G, Reynier P, Bonneau D, Marques W, Jr., Lenaers G, McFarland R, Taylor RW, Turnbull DM, Votruba M, Zeviani M, Carelli V, Bindoff LA, Horvath R, Amati-Bonneau P, and Chinnery PF. 2010. Multi-system neurological disease is common in patients with OPA1 mutations. Brain 133:771-786. 10.1093/brain/awq007

Zanna C, Ghelli A, Porcelli AM, Karbowski M, Youle RJ, Schimpf S, Wissinger B, Pinti M, Cossarizza A, Vidoni S, Valentino ML, Rugolo M, and Carelli V. 2008. OPA1 mutations associated with dominant optic atrophy impair oxidative phosphorylation and mitochondrial fusion. Brain 131:352-367. 10.1093/brain/awm335

Zerbino DR, Achuthan P, Akanni W, Amode MR, Barrell D, Bhai J, Billis K, Cummins C, Gall A, Giron CG, Gil L, Gordon L, Haggerty L, Haskell E, Hourlier T, Izuogu OG, Janacek SH, Juettemann T, To JK, Laird MR, Lavidas I, Liu Z, Loveland JE, Maurel T, McLaren W, Moore B, Mudge J, Murphy DN, Newman V, Nuhn M, Ogeh D, Ong CK, Parker A, Patricio M, Riat HS, Schuilenburg H, Sheppard D, Sparrow H, Taylor K, Thormann A, Vullo A, Walts B, Zadissa A, Frankish A, Hunt SE, Kostadima M, Langridge N, Martin 
522

523

524

525

526

527

528

529

530
FJ, Muffato M, Perry E, Ruffier M, Staines DM, Trevanion SJ, Aken BL, Cunningham F, Yates A, and Flicek P. 2018. Ensembl 2018. Nucleic Acids Res 46:D754-D761. $10.1093 / \mathrm{nar} / \mathrm{gkx} 1098$

Zhang J, Liang Y, and Zhang Y. 2011. Atomic-Level Protein Structure Refinement Using Fragment-Guided Molecular Dynamics Conformation Sampling. Structure 19:1784-1795. 10.1016/j.str.2011.09.022

Zhang Y, and Skolnick J. 2005. TM-align: a protein structure alignment algorithm based on the TM-score. Nucleic Acids Res 33:2302-2309. 10.1093/nar/gki524 


\section{Figure Legends}

532 Figure 1: Schematic representation of human OPA1 isoforms. (A) In human, OPAl gene is 533 built from 30 exons, 3 of which $(4,4 b$ and $5 b)$ are alternatively spliced leading to 8 variants 534 (isoforms 1-8). OPA1 exons (numbers) were schematized by short-term. Mitochondrial 535 proteolytic cleavage sites for mitochondrial processing peptidase (MPP), OMA1 (S1) and 536 YME1L (S2) were indicated. (B) Average hydropathicity of OPA1 N-terminal exon-encoded 537 peptides. (C) The pI value of OPA1 N-terminal exon-encoded peptides. Proteolytic cleavage at 538 S1 and S2 sites by the OMA1 and YME1L peptidase generates the IMS soluble s-forms. Exon 5539 encoded peptide was divided into two parts by site S1, while the N-terminal part was labeled 540 with $5^{\mathrm{N}}$ and the C-terminal was labeled with $5^{\mathrm{C}}$. The same labeling method was used for exon $5 \mathrm{~b}-$ 541 encoded peptide cleaved at site S2.

542 Figure 2: The three-dimensional structure of human OPA1 protein. (A) Structure-based

543 domain architecture of OPA1 isoform 8. The domain assignment was indicated below. Each 544 domain was labeled by different colors, corresponding to the schematic representation of exons. 545 (B) Modeled structure of isoform 8 excluding MTS and TM (long form). It was showed in a 546 cartoon mode. The N-terminal region was rich in alpha helix and did not include specific 547 domains, while The C-terminal region of OPA1 protein was a dense structure containing 548 multiple domains. (C) Modeled structure of human OPA1 protein (short form). It was displayed 549 in a cartoon mode. 
550

551

552

553

554

555

556

557

558

559

560

561

562

563

564

565

566

567

568

569

Figure 3: Comparison between the structure of OPA1 protein and other human dynamins.

(A) Phylogenetic tree of human dynamin superfamily members. The structure of OPA1 protein was compared with that of dynamin 1 (B), dynamin 3 (C), Drp1 (D), MxA (E), MxB (F), mitofusin $1(\mathrm{G})$, GBP1 (H), atlastin 1 (I) and atlastin 3 (J) separately. Phylogenetic analyses were conducted by the bootstrap neighbor joining method. The tree was drawn to scale, with branch lengths in the same units as those of the evolutionary distances used to infer the phylogenetic tree.

The structure of OPA1 was showed by modeled structure, while dynamin 1, dynamin 3, Drp1, MxA, MxB, mitofusin 1, GBP1, atlastin 1 and atlastin 3 were displayed by crystal structures (PDB code: 3snh, 5a3f, 4bej, 3szr, 4whj, 5yew, 1dg3, 3q5e and 5vgr). The TM-score calculated according to the comparison results was also displayed.

Figure 4: Prediction of OPA1 homodimeric interaction. (A) One stable mode of dimerization. The homodimerization interface had been rotated. (B) The other possible mode of dimerization. Cartoon figure of OPA1 homodimeric interaction predicted through docking calculations. The right was inter-residues interaction in potential dimerization interfaces. The interaction partners were connected by broken lines. The modeled structure of OPA1 C-terminal region was used for docking analysis. The residues were numbered according to the human OPA1 isoform 8 .

Figure 5: Evolutionary conservation of vertebrates OPA1 protein sequence. (A) Mapping of evolutionary conservation of amino acid positions in the OPA1. The conservation scale was defined from the most variable amino acid positions (grade 1, colored turquoise) which were considered as rapidly evolving, to the most conservative positions (grade 9, colored maroon) 
570 which were considered as slowly evolving. The nine-color conservation grades were mapped

571 onto the structure of human OPA1. (B) Distribution of evolutionary conservation in human

572 OPA1 gene according to the exons. The highest percent of maroon represented the most

573 conserved position in a protein. 


\section{Figure 1}

Figure 1: Schematic representation of human OPA1 isoforms.

(A) In human, OPA1 gene is built from 30 exons, 3 of which (4, 4b and 5b) are alternatively spliced leading to 8 variants (isoforms 1-8). OPA1 exons (numbers) were schematized by short-term. Mitochondrial proteolytic cleavage sites for mitochondrial processing peptidase (MPP), OMA1 (S1) and YME1L (S2) were indicated. (B) Average hydropathicity of OPA1 Nterminal exon-encoded peptides. (C) The pl value of OPA1 N-terminal exon-encoded peptides. Proteolytic cleavage at $\mathrm{S} 1$ and $\mathrm{S} 2$ sites by the OMA1 and YME1L peptidase generates the IMS soluble s-forms. Exon 5 -encoded peptide was divided into two parts by site $\mathrm{S} 1$, while the $\mathrm{N}$ terminal part was labeled with $5^{N}$ and the C-terminal was labeled with $5^{\complement}$. The same labeling method was used for exon 5b-encoded peptide cleaved at site S2. 


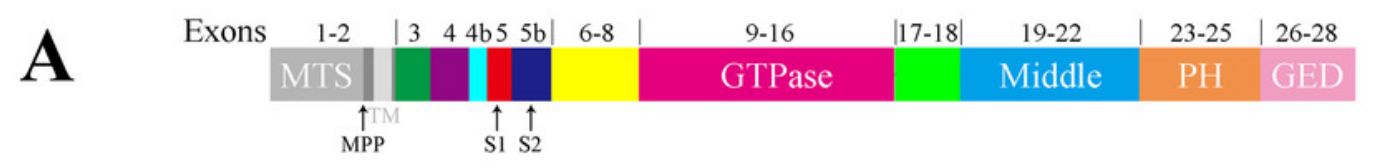

\begin{tabular}{|c|c|c|c|c|c|c|}
\hline Isoform 1 & MTS & GTPase & Middle & PH & GED & 960 aа \\
\hline Isoform 2 & MTS & GTPase & Middle & PH & GED & 924 aа \\
\hline Isoform 3 & MTS & GTPase & Middle & PH & GED & 942 aа \\
\hline Isoform 4 & MTS & GTPase & Middle & PH & GED & 961 aа \\
\hline Isoform 5 & MTS & GTPase & Middle & PH & GED & 978 aа \\
\hline Isoform 6 & MTS & GTPase & Middle & PH & GED & 979 аа \\
\hline Isoform 7 & MTS & GTPase & Middle & PH & GED & 997 аа \\
\hline Isoform 8 & MTS & GTPase & Middle & PH & GED & 1015 aа \\
\hline
\end{tabular}
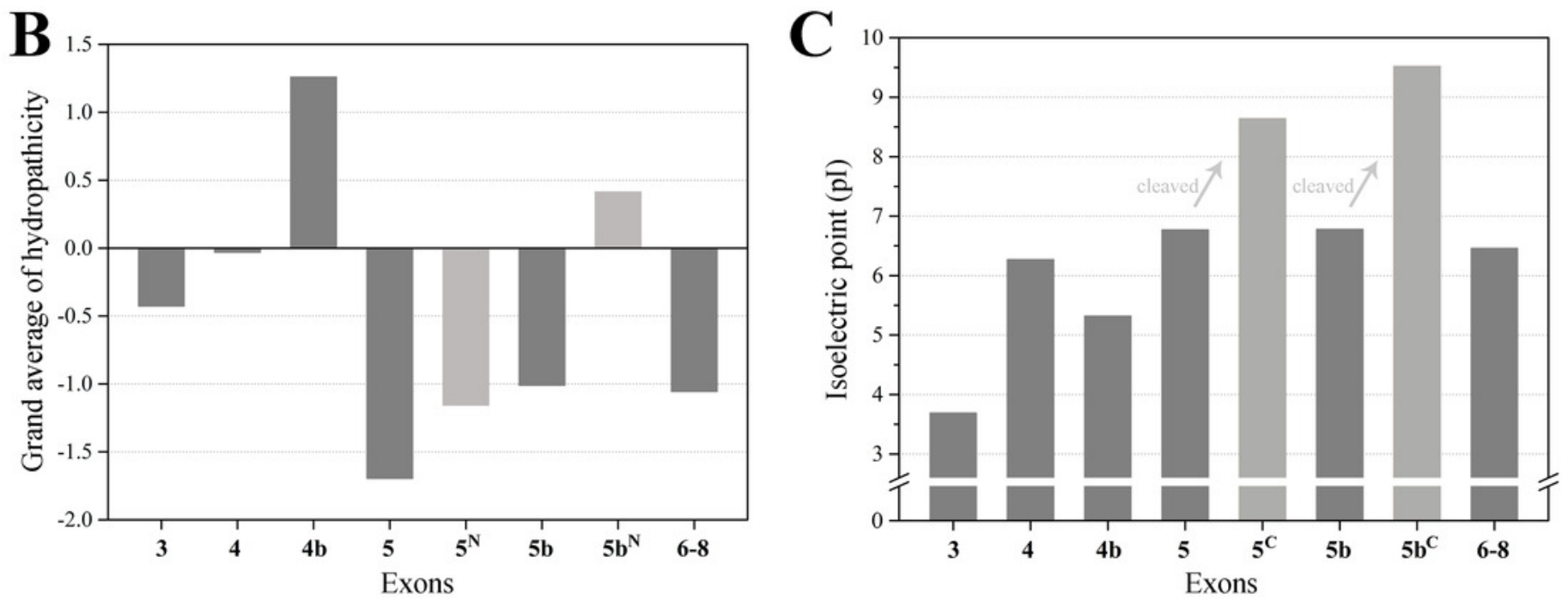


\section{Figure 2}

Figure 2: The three-dimensional structure of human OPA1 protein.

(A) Structure-based domain architecture of OPA1 isoform 8. The domain assignment was indicated below. Each domain was labeled by different colors, corresponding to the schematic representation of exons. (B) Modeled structure of isoform 8 excluding MTS and TM (long form). It was showed in a cartoon mode. The $\mathrm{N}$-terminal region was rich in alpha helix and did not include specific domains, while The C-terminal region of OPA1 protein was a dense structure containing multiple domains. (C) Modeled structure of human OPA1 protein (short form). It was displayed in a cartoon mode.

A

Exons

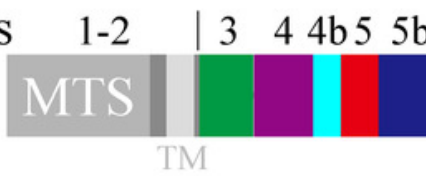

6-8

9-16

|17-18

GTPase

$19-22$

\begin{tabular}{l|l}
$23-25$ & $26-28$
\end{tabular}

Middle PH GED

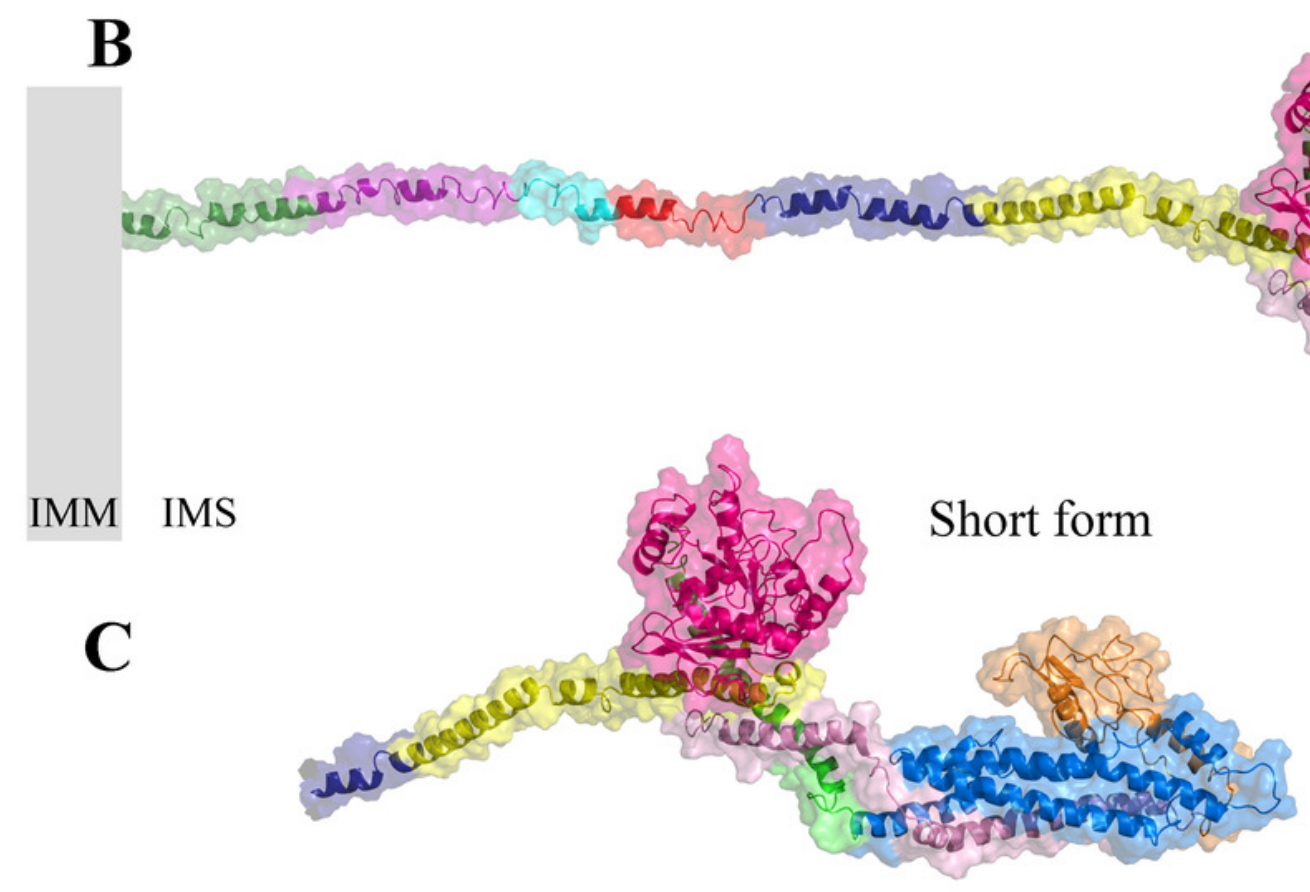

Long form

IMM IMS

Short form

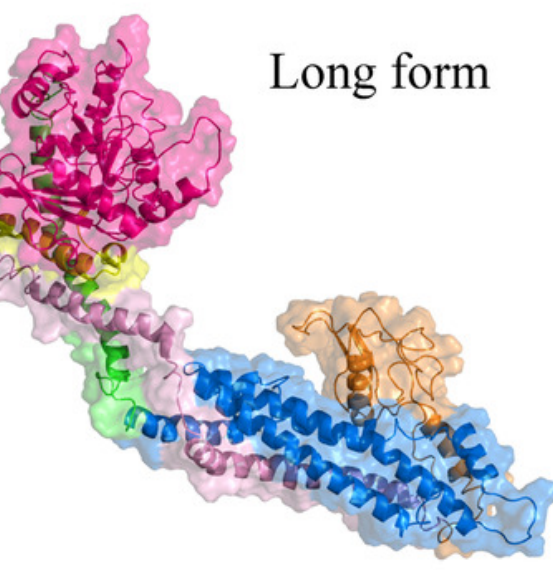




\section{Figure 3}

Figure 3: Comparison between the structure of OPA1 protein and other human dynamins.

(A) Phylogenetic tree of human dynamin superfamily members. The structure of OPA1 protein was compared with that of dynamin 1 (B), dynamin 3 (C), Drp1 (D), MxA (E), MxB (F), mitofusin $1(\mathrm{G}), \mathrm{GBP} 1(\mathrm{H})$, atlastin 1 (I) and atlastin $3(\mathrm{~J})$ separately. Phylogenetic analyses were conducted by the bootstrap neighbor joining method. The tree was drawn to scale, with branch lengths in the same units as those of the evolutionary distances used to infer the phylogenetic tree. The structure of OPA1 was showed by modeled structure, while dynamin 1, dynamin 3, Drp1, MxA, MxB, mitofusin 1, GBP1, atlastin 1 and atlastin 3 were displayed by crystal structures (PDB code: 3snh, 5a3f, 4bej, 3szr, 4whj, 5yew, 1dg3, 3q5e and 5vgr). The TM-score calculated according to the comparison results was also displayed. 
A

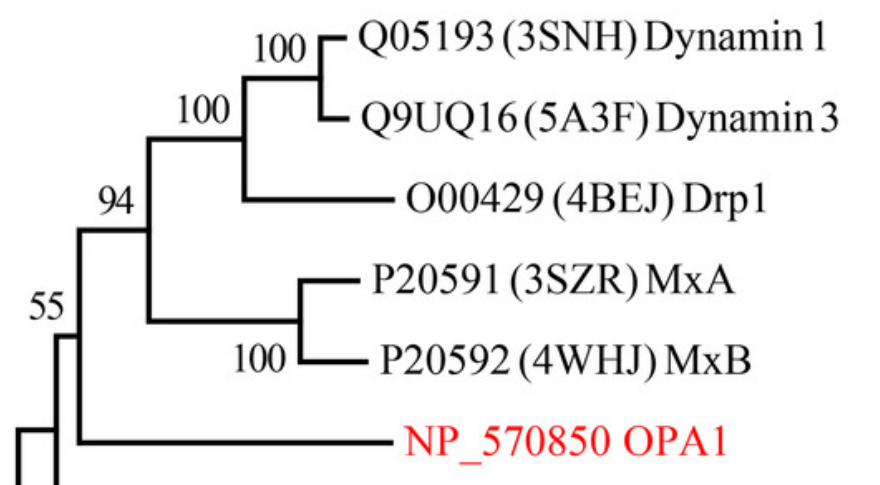

Q8IWA4(5YEW) Mitofusin 1

P32455 (1DG3) GBP1 Q8WXF7 (3Q5E) Atlastin 1 Q6DD88(5VGR)Atlastin 3
TM-score

0.86

0.78

0.60

0.52

0.63

0.30

0.24

0.26

0.26
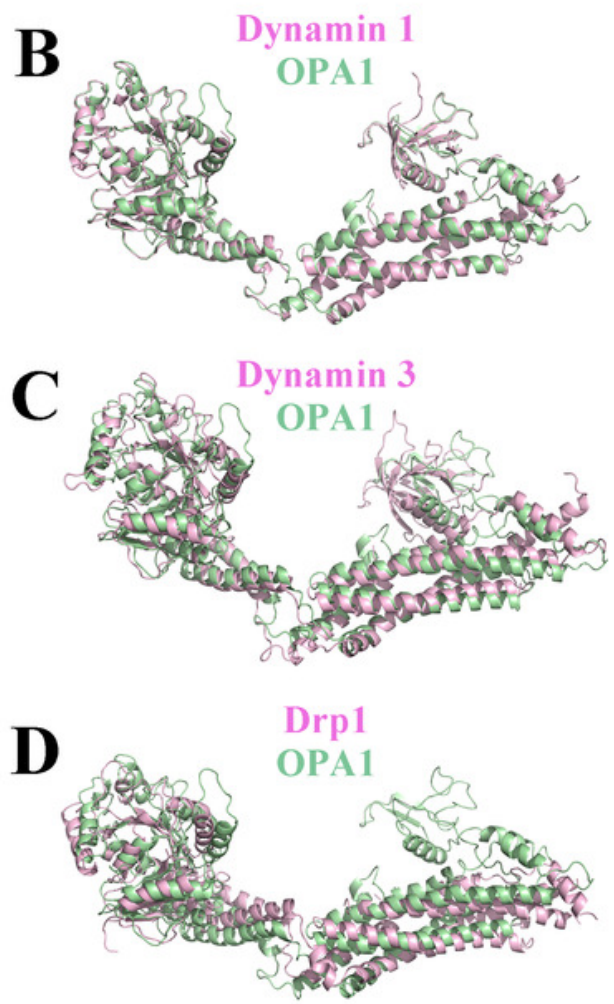

$\mathbf{E}$

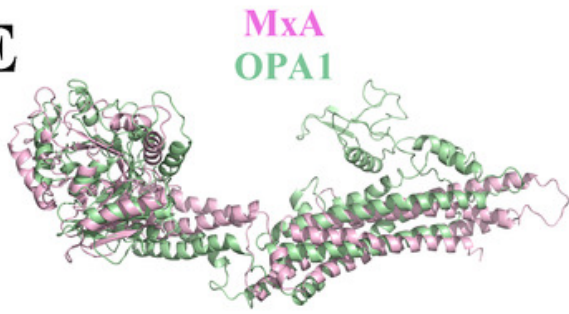

$\mathbf{H}$

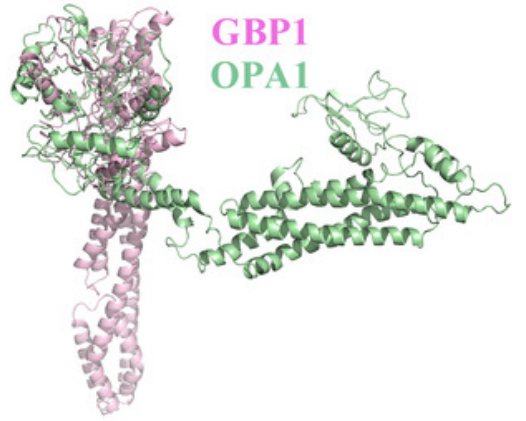

Atlastin 1

OPA1

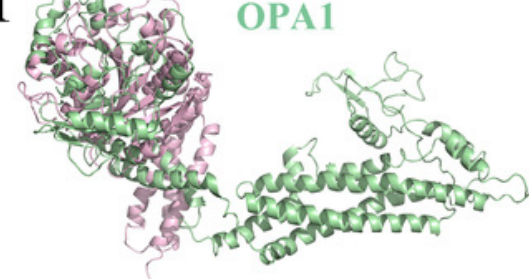

$\mathbf{J}$

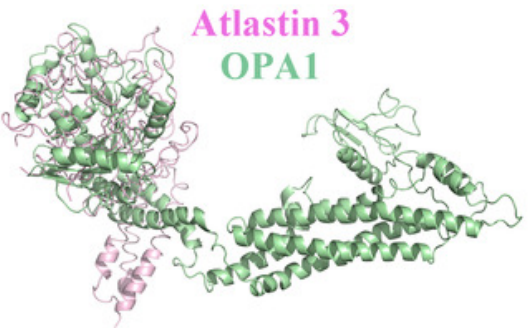

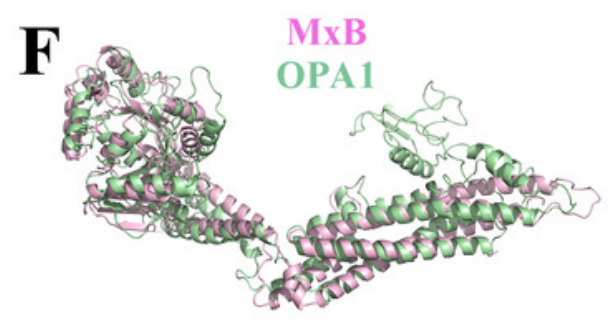




\section{Figure 4}

Figure 4: Prediction of OPA1 homodimeric interaction.

(A) One stable mode of dimerization. The homodimerization interface had been rotated. (B) The other possible mode of dimerization. Cartoon figure of OPA1 homodimeric interaction predicted through docking calculations. The right was inter-residues interaction in potential dimerization interfaces. The interaction partners were connected by broken lines. The modeled structure of OPA1 C-terminal region was used for docking analysis. The residues were numbered according to the human OPA1 isoform $8 . "$ 
A
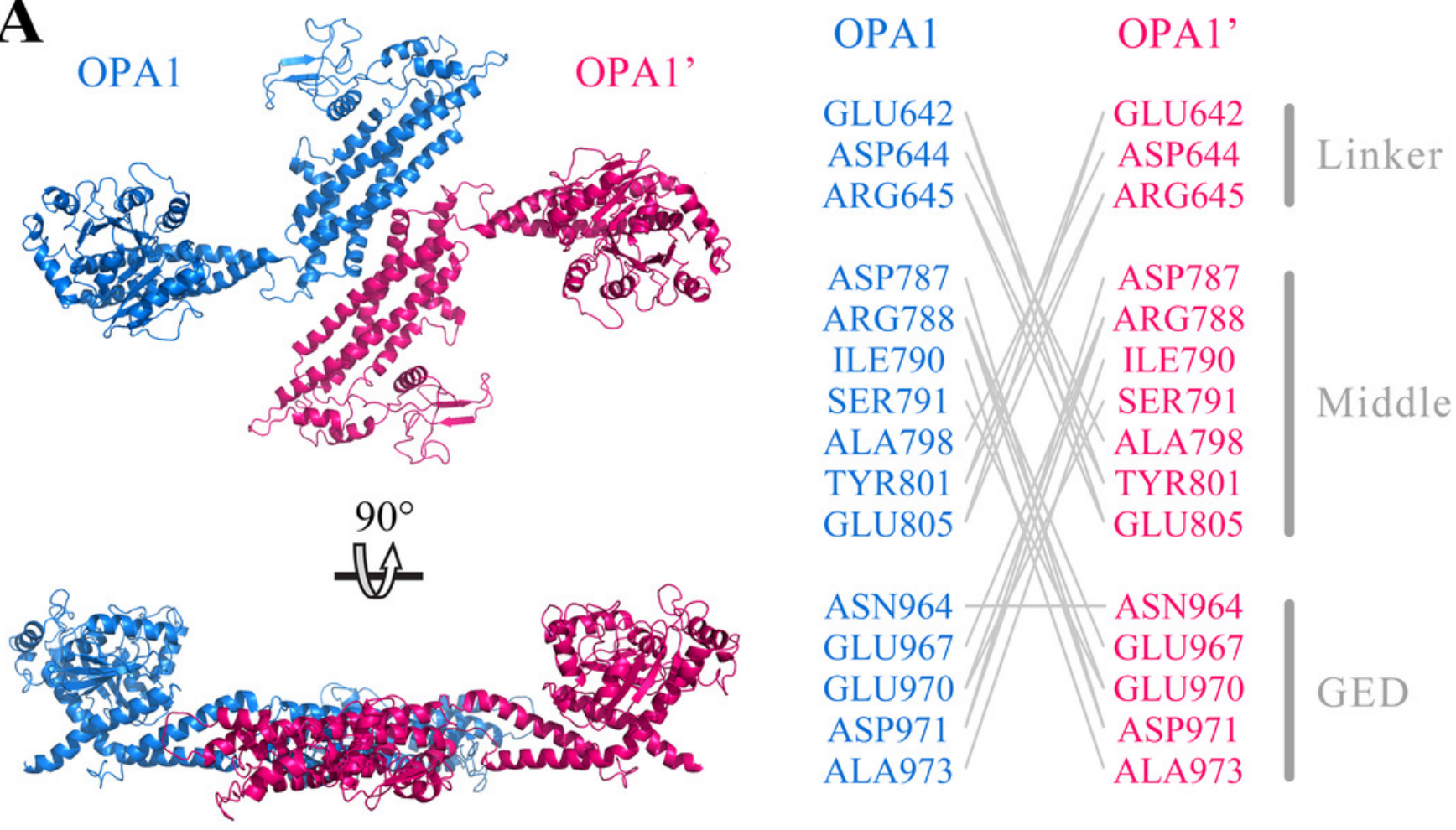

B
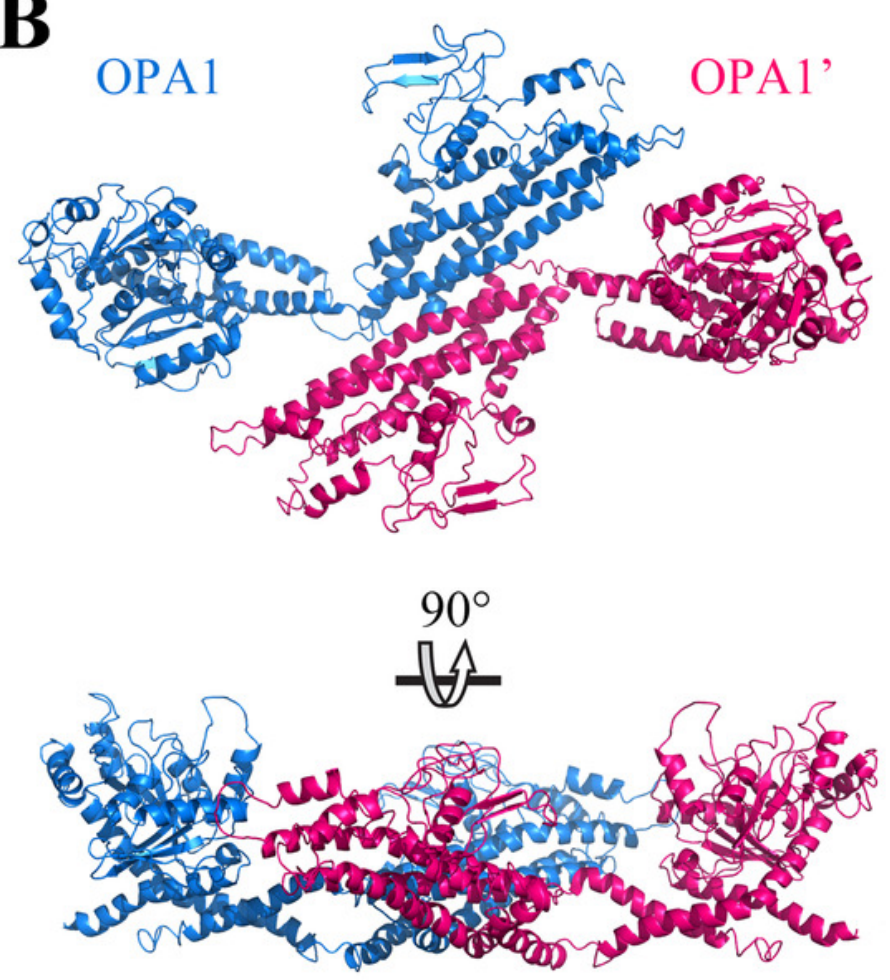

\begin{tabular}{c|c} 
OPA1 & OPA1' \\
PHE772 \\
SER776 \\
VAL779 \\
ILE780 \\
ASN783 \\
SER776 \\
ALA784 \\
ASP77779 \\
ILE780 \\
ARG788 \\
ILE790 \\
SER791 \\
GLN793 \\
ALA784 \\
THR956 \\
ASN964 \\
ASP787 \\
ASP971 \\
PHE972 \\
ALA973 \\
ASP975 \\
ILE790 \\
GLU977
\end{tabular}




\section{Figure 5}

Figure 5: Evolutionary conservation of vertebrates OPA1 protein sequence.

(A) Mapping of evolutionary conservation of amino acid positions in the OPA1. The conservation scale was defined from the most variable amino acid positions (grade 1, colored turquoise) which were considered as rapidly evolving, to the most conservative positions (grade 9, colored maroon) which were considered as slowly evolving. The nine-color conservation grades were mapped onto the structure of human OPA1. (B) Distribution of evolutionary conservation in human OPAl gene according to the exons. The highest percent of maroon represented the most conserved position in a protein. 
A

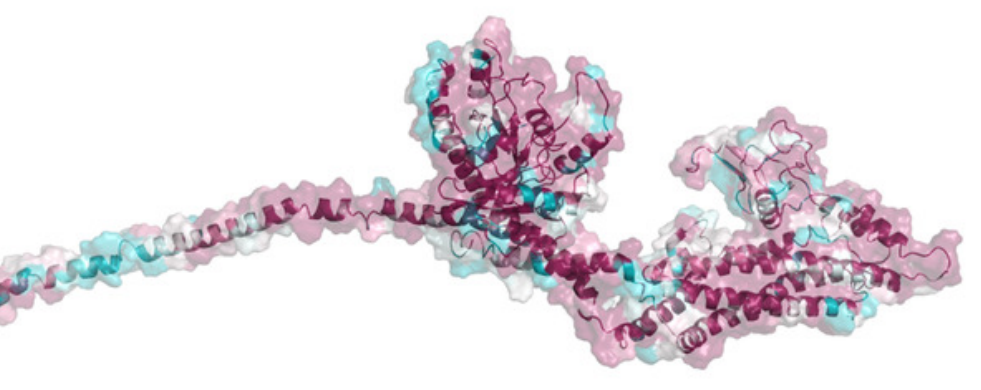

Variable

Conserved

\begin{tabular}{|l|llllllll|l|}
1 & 2 & 3 & 4 & 5 & 6 & 7 & 8 & 9 \\
\hline
\end{tabular}

B

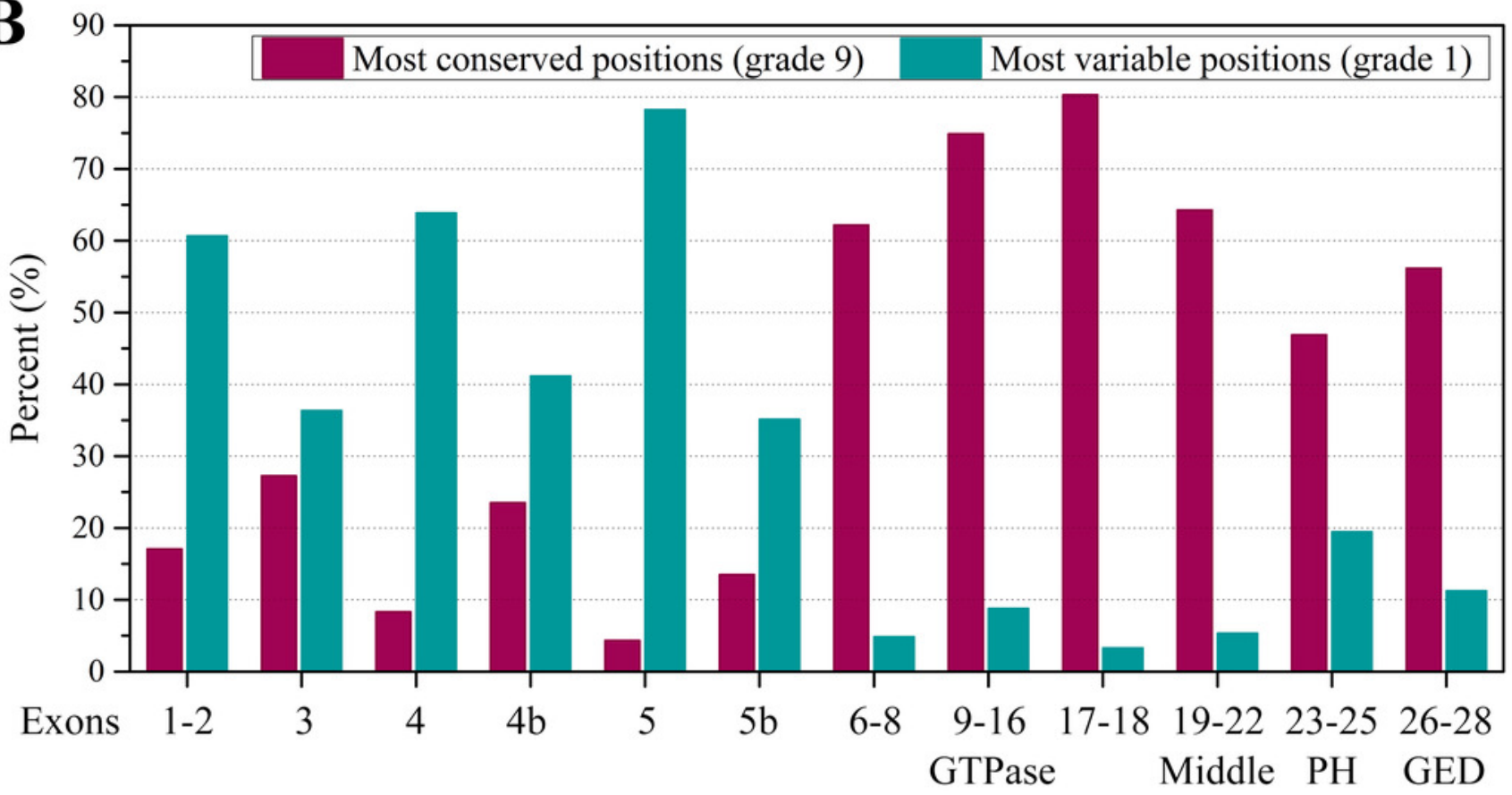

